\title{
Die Corona-Krise im Diskurs: Hervorbringung, Konzeptualisierung und Vermittlung im internationalen Vergleich
}

\author{
Anne-Laure Daux-Combaudon · Stephan Habscheid • Sandra Herling • \\ Britta Thörle
}

Angenommen: 13. September 2021 / Online publiziert: 6. Oktober 2021

(C) Springer-Verlag GmbH Deutschland, ein Teil von Springer Nature 2021

Am 1. Mai 2020 sagte der Historiker Adam Tooze im Zusammenhang mit der COVID-19-Pandemie in einem Interview mit der Zeitung Le Monde: »Noch nie hat die Welt einen so gewaltigen Schock erlebt, in einer solchen Größenordnung und in einem Ausmaß, das von Tag zu Tag vor unseren Augen bedrohlicher wird « (Tooze 2020). Im Titel des Artikels heißt es: »Das Ausmaß der Krise wird vor unseren Augen von Tag zu Tag bedrohlicher«. Das Zitat und der Titel des Artikels erinnern an frühere auf Krisen unterschiedlichster Art bezogene Formulierungen wie »die Krise ist da, sie ist brutal, sie ist schwer, sie ist tief, sie ist hart « (zit. nach Osthus 2016), »wie konnte es so weit kommen? «(Durand/Lefèvre/Prak-Derrington 2017) oder die für Krisendiskurse typische Formel »noch nie zuvor« (zit. nach Wengeler 2010). Krisen als solche hängen also offensichtlich auch von sprachlichen Voraussetzungen ab, werden mit Hilfe von Sprache kommunikativ konstituiert. Vor diesem Hintergrund hat auch die Linguistik einen Beitrag zur Erklärung von Krisen und ihrer jeweiligen Dynamik zu leisten.

\footnotetext{
Anne-Laure Daux-Combaudon $(\bowtie)$

Angewandte Fremdsprachen, Université Sorbonne Nouvelle, Paris, Frankreich

E-Mail: anne-laure.daux@ sorbonne-nouvelle.fr

Stephan Habscheid

Germanistik, Universität Siegen, Siegen, Deutschland

E-Mail: habscheid@germanistik.uni-siegen.de

Sandra Herling · Britta Thörle

Romanisches Seminar, Universität Siegen, Siegen, Deutschland

Sandra Herling

E-Mail: herling@ romanistik.uni-siegen.de

Britta Thörle

E-Mail: thoerle@romanistik.uni-siegen.de
} 
Die sprachwissenschaftliche Beschäftigung mit Krisendiskursen ist nicht neu und hat sich u.a. ausgehend von der Finanzkrise 2008 als Forschungsfeld weiterentwickelt. Unter anderem wurden bestimmte Konstanten von Krisendiskursen herausgearbeitet, etwa ihr gemeinsames »Basisvokabular« (Wengeler/Ziem 2014) oder die wiederkehrenden sprachlich-kommunikativen Ressourcen der Krisenkonstitution im medialen Diskurs, darunter der Rekurs auf narrative Strukturen und tradierte Geschichten (vgl. zum Wirtschaftskontext Kleeberg 2009), typische Metaphern sowie die Verwendung bestimmter Argumentationsmuster und Topoi (Nünning 2012; Wengeler/Ziem 2014; Habscheid/Koch 2014).

Mit dem Ausbruch der COVID-19-Pandemie hat die sprachwissenschaftliche Beschäftigung mit Krisendiskursen erneut Fahrt aufgenommen. Schon in der ersten Jahreshälfte 2020 erschienen erste Publikationen, wurden Kolloquien und Workshops veranstaltet, die die Pandemie »in Echtzeit« (Volkmer/Werner 2020, S. 12) analysierten (vgl. auch das von Roth/Wengeler 2020 herausgegebene Corona-Themenheft der Zeitschrift Aptum mit einer größeren Zahl »essayistischer Notizen zum Diskurs«). Dabei ist die Rolle von Sprache und Kommunikation in der Pandemie nicht nur Gegenstand wissenschaftlicher Reflexion, sondern weckte schon früh auch das Interesse einer breiten Öffentlichkeit. Zu beobachten ist dies u.a. anhand von populären Glossaren zu Neologismen und Sprachchroniken (für eine Übersicht über deutschsprachige Glossare siehe z.B. Möhrs 2020; zum Französischen und weiteren Sprachen die Sammlung von Pressetexten zur Rubrik »Les mots du virus « der Bibliotheken der Universität Aix-Marseille ${ }^{1}$; zum französisch-deutschen Vergleich Balnat 2020), aber auch in den (teilweise als populistisch bewerteten) öffentlichen Debatten um die Angemessenheit und Glaubwürdigkeit politischer und wissenschaftlicher Krisenbeschreibungen.

Die in diesem Band versammelten Beiträge beschäftigen sich mit der CoronaKrise nun bereits (trotz ihres Andauerns) mit etwas größerem zeitlichen Abstand. Sie analysieren die Krise konsequent unter dem Aspekt ihrer sprachlichen Konstitution, über verschiedene institutionelle Kontexte hinweg sowie im Länder- und Sprachenvergleich. Indem die krisenhafte Wirklichkeit kommunikativ als solche benannt, erklärt, als praktische Herausforderung thematisiert oder bestritten wird, wird die Krise jeweils auch sprachlich etabliert, geformt, charakterisiert und verhandelbar gemacht: Wie das von Veniard (2013) für das Französische erstellte lexikodiskursive Profil zeigt, impliziert beispielsweise die Verwendung des Wortes crise selbst die soziale Sinnzuschreibung für ein Ereignis als »Einschnitt« ([+rupture $]$ ), »Phase « ([+période $])$, »schwerwiegend « ([+intensité]) und »überwindungsbedürftig « ([+dont on veut voir arriver le terme $])$ - das Reden von der »Krise« hat damit zugleich Diagnose- und Appellcharakter (vgl. auch Habscheid/Koch 2014, S. 8).

Ein entsprechender Einschnitt durch die Corona-Krise manifestiert sich im öffentlichen medialen Diskurs in den meisten europäischen Ländern spätestens Mitte März 2020, als - anknüpfend an die WHO - von staatlicher Seite die Pandemie offiziell festgestellt wird und weitreichende Kontaktbeschränkungen und damit massive Einschränkungen des öffentlichen und privaten Lebens eingeführt werden. Konstituiert werden die Maßnahmen durch offizielle rechtliche und administrative Krisenkom-

${ }^{1}$ URL: https://bu.univ-amu.libguides.com/c.php?g=679422\&p=4842726 [07.09.2021]. 
munikation über alle Ebenen des politischen Systems hinweg. Notwendigerweise begleitet werden sie in den Demokratien von politischer Krisenkommunikation in Parlamenten und Medien, in deren Rahmen Regierungsvertreter_innen das pandemische Geschehen deuten, Maßnahmen legitimieren, emotionale Folgen kommunikativ bearbeiten und die Bevölkerung zur Mitwirkung aufrufen (vgl. Spieß, in diesem Heft). Dabei signalisieren nicht nur Inhalte und Rhetorik, sondern - wie im Falle der Fernsehansprache Angela Merkels am 18.3.2020 - allein schon die Tatsache, dass sich die Bundeskanzlerin zu diesem Zeitpunkt in dieser Form an die Bürger_innen wendet, eine krisenhafte »Denormalisierung « (Link 2013). Gleichzeitig wird diskursiv eine - nur vorübergehende - andersartige Lage etabliert (vgl. Müller, in diesem Heft), deren Konzepte, Einrichtungen, Bestimmungen, Artefakte usw. im Einzelnen sprachlich benannt und gerechtfertigt werden müssen. Deren konkrete Gestaltung wird zum Gegenstand politisch-sprachlicher Kontroversen.

Kommunikativ konstituiert wird der Verlust von Normalität regelmäßig auch durch die von wissenschaftlichen bzw. staatlichen Einrichtungen bereitgestellten, medial verbreiteten und mit Hilfe von Expert_innen interpretierten Kennzahlen, besonders der Infektions- oder Todesfälle. In den Blick der Medienöffentlichkeit rücken damit Epidemiolog_innen und Virolog_innen, die in Pressekonferenzen, Talkshows und Podcasts ihr Expertenwissen einem breiten Laienpublikum sprachlich vermitteln und deren Aussagen von politischen Entscheidungsträgern zur Legitimierung von Maßnahmen herangezogen oder auch - wie etwa im Fall des brasilianischen Präsidenten Bolsonaro - im Zuge einer Verweigerung politischer Maßnahmen bestritten werden (vgl. Leschzyk, in diesem Heft).

Kommunikativ konstituiert wird die Krise auch im Alltagshandeln, wo u.a. Alltagspraktiken durch den staatlich regulierten Umgang mit körperlicher Nähe und Distanz nun eine potentiell »politisierte Konnotation« erhalten (vgl. Vogel 2020). So kann der Verzicht auf das Tragen einer Maske als politischer Protest inszeniert werden, ebenso wie beispielsweise das Zuhausebleiben oder das Abstandhalten als (rituelle) Symbole für eine »solidarische « Haltung gewertet werden können (Liedtke 2020).

Auf einer Art Zwischenebene zwischen Alltags- und institutionellem Diskurs (vgl. Domke 2014) manifestiert sich die Krise schließlich in den Linguistic Landscapes von Städten (vgl. Behr/Liedtke, in diesem Heft), wo u.a. verschiedenste Formen kleiner Kommunikate (Aushänge, Hinweisschilder, ...) auf pandemiebedingte Geschäftsschließungen, Abstandsregeln, Zugangsbeschränkungen usw. nicht nur hinweisen, sondern diese performativ mit herstellen (Androutsopoulos 2020, vgl. zum performativen Charakter auch Habscheid/Reuther 2013), indem sie das auch körperliche Verhalten im öffentlichen, soziomateriellen Raum regulieren und u.U. zur Etablierung neuer körperlicher Routinen beitragen.

Eine Konstante der öffentlichen Diskurse der Corona-Krise ist paradoxerweise ihre relative thematische Konzentration auf den nationalen Raum. Während die globale Vernetzung durch die ungebremste Verbreitung des Virus in alle Teile der Welt offensichtlich wird, erscheint der Krisendiskurs erstaunlich national geprägt (vgl. Bubenhofer et al. 2020, S. 163, für die Deutschschweiz sowie Knoblauch 2020). Vor diesem Hintergrund kann es interessant sein, Corona-Krisendiskurse länder- und sprachenübergreifend zu betrachten, wie dies in dem vorliegenden Heft geschieht. 
Irmtraud Behr und Frank Liedtke beschäftigen sich mit dem konstitutiven Aspekt der medialen Krisenkommunikation anhand eines vielfältigen Korpus von ortsfesten Kommunikaten, d.h. coronaspezifischen Aushängen, Aufklebern, gesprayten Markierungen, elektronischen Anzeigetafeln und Aufstellern, aus vier europäischen Ländern (Frankreich, Deutschland, Österreich und Schweden), die zwischen März 2020 und März 2021 wahrzunehmen waren. Indem die Kommunikate sich auf die Krise beziehen (sie sind reaktive Wortmeldungen im allgemeinen Corona-Diskurs oder Transkriptionen der beschlossenen öffentlichen Maßnahmen), konstituieren sie sie sprachlich-diskursiv mit. Die Corona-Kommunikate sind Teil der Krise im Sinne der Konstitution von neuen Routinen. Indem sie Raum für verschiedene Arten der Expressivität geben, sind sie aber auch Teil der emotionalen Bewältigung der Krise in dem Sinne, dass sie Ausdrucksmöglichkeiten für Emotionen und Appelle schaffen sowie Solidarität benennen und einfordern. Im Beitrag werden die Kommunikate unter dem Aspekt ihrer Materialität, Medialität und Lokalität sowie der jeweiligen diskursiven Funktionen analysiert. Behr/Liedtke unterscheiden drei Phasen in der Verwendung von Kommunikaten in der Pandemie und sehen in der Kombination Expressivität/Information plus Regulation ein kommunikatives Muster. Eine grundsätzliche Eigenschaft öffentlicher Kommunikate dieser Art ist schließlich ihre Arealität. Unter Arealität werden die im weitesten Sinne räumlichen Eigenschaften der Kommunikate mit den jeweils spezifischen Rezeptions- und Befolgungsbedingungen verstanden. Es wird die Hypothese entwickelt, dass eine Typologie der Kommunikate unter dem Aspekt ihrer Arealität aufgestellt werden kann, wodurch vertiefte Einsichten in ihre sozialen und kommunikativen Funktionen ermöglicht werden.

Im Mittelpunkt der Überlegungen von Sandrine Reboul-Touré steht der Begriff $>$ Krise <: Wie wird z.B. das Corona-Ereignis benannt? Ist es eine Epidemie, eine Pandemie, eine Gesundheitskrise (crise sanitaire) usw.? Reboul-Touré positioniert sich im Rahmen der diskursiven Semantik (sémantique discursive) und analysiert lexikalische Elemente im Diskurs, mit denen sie das Ereignis erfasst. Ihr Korpus besteht aus französischen Pressetexten aus dem Jahr 2020, die von den Datenbanken Factiva und Europress bereitgestellt werden. Ihre Analysen zeigen u.a., dass Krisenerwähnungen im Corona-Kontext stark zunehmen. Einen weiteren Aspekt der Auswirkungen der Krise auf sprachlicher Ebene bilden Wortneubildungen, die sowohl im institutionellen Diskurs (confinement, déconfinement und reconfinement für die aufeinanderfolgenden Lockdowns; septaine, quatorzaine für die Quarantäne, die keine vierzig Tage dauert) als auch im Alltagsdiskurs (vor allem Kofferwörter) zu finden sind. Dabei wird klar, dass und wie die Corona-Krise auch ein sprachliches Ereignis ist.

Marcus Müller richtet auf korpuslinguistischer Grundlage sein Augenmerk besonders auf die Funktionen von Modalverben, deren angemessenen Gebrauch er als einen besonders wichtigen Faktor erfolgreicher Krisenkommunikation empirisch nachzuweisen versucht. Als Beispiel dient die Krisenkommunikation in der Bundesrepublik Deutschland in der ersten Phase der Pandemie bis Ende März 2020. Distributiv quantifiziert und diskurspragmatisch analysiert werden insbesondere die verschiedenartigen Redehintergründe und ihre individuellen Ausprägungen im Corona-Krisendiskurs. Müller zeigt unter anderem auf, dass und wie die mit Hilfe von Modalverben aufgerufenen normativen Redehintergründe nicht nur auf behördliche 
Maßnahmen und deren Effekte verweisen, sondern auch auf soziale Normen, deren Geltung als selbstverständlich vorausgesetzt wird. Teleologische Redehintergründe, die mit der Verwendung von Modalverben einhergehen, bringen nach Müller eine Perspektive der Re-Normalisierung als Korrektiv zur Krisenkommunikation ins Spiel.

Constanze Spieß widmet sich in ihrem Beitrag einem Vergleich der CoronaKrisenkommunikation des österreichischen Bundeskanzlers Sebastian Kurz und der deutschen Bundeskanzlerin Angela Merkel. Dazu werden Regierungserklärungen und Ansprachen an die Bevölkerung in verschiedenen Medien im Zeitraum zwischen März 2020 und März 2021 als Kommunikationsformen typologisiert und diskurslinguistisch (im Blick auf topische Muster, Redehandlungen und Adressierungen/ Positionierungen sowie Strategien der politischen Kommunikation) untersucht. Neben diversen situationsbedingten Gemeinsamkeiten werden relevante Unterschiede herausgearbeitet, vor allem im Blick auf Kurz die identitätspolitische Selbstaufwertung Österreichs und der Österreicher in Verbindung mit der Abwertung bzw. Ausgrenzung >anderer $<$ nach außen und im Inneren.

In ihrem Beitrag richtet Dinah Leschzyk das Interesse auf das Phänomen der Infodemie und der damit für die Krisenkommunikation grundlegenden Frage, inwiefern Glaubwürdigkeit erzeugt oder auch untergraben werden kann. Im Mittelpunkt der kontrastiv ausgerichteten Untersuchung stehen Äußerungen, die seitens des brasilianischen Präsidenten Jair Messias Bolsonaro und Politikern der Partei AfD (Alternative für Deutschland) im Kontext der COVID-19-Pandemie auf Twitter im ersten Jahr der Pandemie gepostet wurden. Die Analyse zeigt unterschiedliche diskursive Strategien der jeweiligen politischen Akteure auf: Während Bolsonaros Rhetorik auf die Unglaubwürdigkeit brasilianischer Medien abzielt, indem z.B. die Verbreitung von Falschmeldungen unterstellt wird, konzentriert sich die Kritik der Partei AfD auf die Kommunikation von Bund und Ländern. In Bezug auf die aristotelischen Kriterien zur Glaubwürdigkeit (gesunder Menschenverstand, moralischer Charakter und guter Wille), die Leschzyk u.a. als theoretischen Rahmen der Studie heranzieht, kann festgehalten werden, dass die AfD sich auf das erstgenannte Kriterium fokussiert und den >mangelnden Sachverstand < der Regierung betont. Im Gegensatz dazu konzentriert sich Bolsonaro auf den nicht-moralischen >Charakter< der Medien. Beide Strategien lassen Institutionen wie auch Medien als unzuverlässig und Misstrauen erweckend im gegenwärtigen Pandemie-Diskurs erscheinen.

Stephan Habscheids und Friedemann Vogels Beitrag widmet sich der Corona-Krisenkommunikation von Bürgermeister_innen. Die Autoren gehen davon aus, dass die Bürgermeister_innen von einer zweifachen Krise betroffen sind: von der Corona-Pandemie und von der Infragestellung der repräsentativen Demokratie durch als »populistisch« bezeichnete politische Akteure. Untersucht wird zum einen, wie Bürgermeister_innen in dieser zweifachen Krise in den Sozialen Medien mit den Bürger_innen interagieren, und zum anderen, inwiefern sich das im öffentlichen Diskurs vermittelte Bild von Bürgermeister_innen in der Corona-Krise verändert. Die praxeologische Auswertung der Facebook-Seite eines Oberbürgermeisters lenkt den Blick auf Verfahren der Krisenbearbeitung in verschiedenen Aufgabenfeldern der Bürgermeisterkommunikation und auf die Kommentarlisten, in denen sich interaktive Klärungs- und Aushandlungsprozesse an die Beiträge des Bürgermeisters 
anschließen. Die korpuslinguistische Untersuchung der Darstellung von Bürgermeister_innen in Pressetexten zeigt u.a. eine gewisse Akzentverschiebung des Bildes von Bürgermeister_innen, das in der Pandemie vor allem durch die Ausübung hoheitlicher Verwaltungsaufgaben geprägt ist, während (medial modifizierte) Aktivitäten aus dem Aufgabenfeld der sozialen Integration seltener thematisiert werden als im Vor-Pandemie-Zeitraum.

Christian Koch und Britta Thörle untersuchen die Corona-Lageberichte in den Pressekonferenzen deutscher und französischer Gesundheitsinstitutionen (Robert Koch-Institut und Direction générale de la santé) im Zeitraum von Mitte März bis Anfang April 2020. Die Analyse der mündlich gehaltenen Expertenvorträge konzentriert sich zunächst auf die Sachverhalts- und Handlungskonstitution und die Frage, wie die Lage von den Akteuren kommunikativ vermittelt wird. Auch die neu entstehende Lexik zur Bezeichnung des Virus und der Masken und der mit den Nominationen verbundene konzeptuelle Wandel werden analysiert. Durch die Gegenüberstellung von deutschen und französischen Textauszügen wird gezeigt, dass die Pressekonferenzen in beiden Ländern viele Gemeinsamkeiten u.a. in Bezug auf die in ihnen realisierten kommunikativen Handlungen und Argumentationsmuster sowie hinsichtlich der Verwendung bestimmter Metaphern und Intensivierungsverfahren aufweisen. Die ermittelten Unterschiede führen Koch/Thörle auf die divergierende politische Funktion der beiden untersuchten Institutionen bzw. auf die unterschiedliche Rollenkonstitution der beteiligten Akteure zurück.

\section{Literatur}

Androutsopoulus, Jannis (2020): Die Sprachlandschaft im Dispositiv der Pandemie. In: Aptum. Zeitschrift für Sprachkritik und Sprachkultur 16/2 + 3, S. 290-299.

Balnat, Vincent (2020): Unter Beobachtung: Corona-Wortschatz im Deutschen und Französischen. In: Nouveaux Cahiers d'Allemand 38/2, S. 139-159.

Bubenhofer, Noah/Knuchel, Daniel/Sutter, Livia/Kellenberger, Maaike/Bodenmann, Niclas (2020): Von Grenzen und Welten: Eine korpuspragmatische COVID-19-Diskursanalyse. In: Aptum. Zeitschrift für Sprachkritik und Sprachkultur 16/2+3, S. 156-165.

Domke, Christine (2014): Die Betextung des öffentlichen Raumes. Eine Studie zur Spezifik von MesoKommunikation am Beispiel von Bahnhöfen, Innenstädten und Flughäfen. Heidelberg: Winter.

Durand, Marie-Laure/Lefèvre, Michel/Prak-Derrington, Emmanuelle (2017): Crises et catastrophes. De la mise en discours à l'argumentation. Cahiers d'études germaniques 73.

Habscheid, Stephan/Koch, Lars (2014): Einleitung: Katastrophen, Krisen, Störungen. In: Zeitschrift für Literaturwissenschaft und Linguistik 44/1, S. 5-12.

Habscheid, Stephan/Reuther, Nadine (2013): Performatisierung und Verräumlichung von Diskursen. Zur soziomateriellen Herstellung von >Sicherheit< an öffentlichen Orten. In: Ekkehard Felder (Hg.): Faktizitätsherstellung in Diskursen. Die Macht des Deklarativen. Berlin/New York: De Gruyter, S. 127-145 (= Sprache und Wissen 13).

Kleeberg, Bernhard (2009): Gewinn maximieren, Gleichgewicht modellieren. In: Christian Klein/Matías Martínez (Hg.) (2009): Wirklichkeitserzählungen. Felder, Formen und Funktionen nicht-literarischen Erzählens. Stuttgart: Metzler, S. 136-159.

Knoblauch, Hubert (2020): Symbole und Räume - Soziologische Reflexionen aus dem Inneren der CoronaKrise. Working Paper No.5. Berlin: SFB 1265.

Liedtke, Frank (2020): Wirus oder: Was es heißt, solidarisch zu sein. In: Aptum. Zeitschrift für Sprachkritik und Sprachkultur 16/2+3, S. 134-141.

Link, Jürgen (2013): Normale Krisen? Normalismus und die Krise der Gegenwart. Konstanz: Konstanz Univ. Press.

Möhrs, Christine (2020): Grübelst du noch oder weißt du es schon? - Glossare erklären Corona-Schlüsselbegriffe. In: Sprachreport 36/3, S. 30-37. https://doi.org/10.14618/sr-3-2020-moe. 
Nünning, Ansgar (2012): Making Crises and Catastrophes: How Metaphors and Narratives Shape Their Cultural Life. In: Carsten Meiner/Kristin Veel (Hg.): The Cultural Life of Catastrophes and Crises. Berlin/Boston: De Gruyter, S. 59-88.

Osthus, Dietmar (2016): »La crise (...) est là, elle est violente, elle est lourde, elle est profonde, elle est dure«. Lexicométrie et narratifs de la crise financière. In: Daniela Pietrini/Katrin Wenz (Hg.): Dire la crise: mots, textes, discours: approches linguistiques à la notion de crise. Frankfurt am Main: Lang, S. $33-48$

Roth, Kersten Sven/Wengeler, Martin (Hg.) (2020): Themenheft: Corona. Essayistische Notizen zum Diskurs. In: Aptum. Zeitschrift für Sprachkritik und Sprachkultur 16/2+3.

Tooze, Adam (2020): L'ampleur de la crise s'aggrave de jour en jour, sous nos yeux. In: Le Monde, 01.05.2020. https://www.lemonde.fr/economie/article/2020/05/01/adam-tooze-1-ampleur-de-lacrise-s-aggrave-de-jour-en-jour-sous-nos-yeux_6038335_3234.html [08.09.2021]

Veniard, Marie (2013): Du profil lexico-discursif de crise à la construction du sens social d'un événement. In: Danielle Londei/Sophie Moirand/Sandrine Reboul-Touré/Licia Reggiani (Hg.): Dire l'événement. Langage, mémoire, société. Paris: Presses Sorbonne Nouvelle, S. 221-232.

Vogel, Friedemann (2020): »Wenn Virologen alle paar Tage ihre Meinung ändern, müssen wir in der Politik dagegenhalten « - Thesen zur politischen Sprache und (strategischen) Kommunikation im PandemieKrisendiskurs. In: Sprachreport 36/3, S. 20-29. https://doi.org/10.14618/sr-3-2020-vog.

Volkmer, Michael/Werner, Karin (Hg.) (2020): Die Corona-Gesellschaft. Analysen zur Lage und Perspektiven für die Zukunft. Bielefeld: transcript.

Wengeler, Martin (2010): >Noch nie zuvor<. Zur sprachlichen Konstruktion der Wirtschaftskrise 2008/2009 im SPIEGEL. In: Aptum. Zeitschrift für Sprachkritik und Sprachkultur 2, S. 138-156.

Wengeler, Martin/Ziem, Alexander (2014): Wie über Krisen geredet wird. Einige Ergebnisse eines diskursgeschichtlichen Forschungsprojekts. In: Zeitschrift für Literaturwissenschaft und Linguistik 44/1, S. 52-74. 